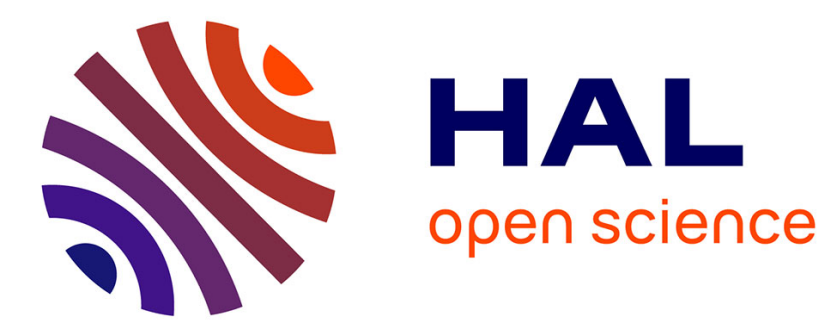

\title{
Fokker-Planck equation for the energy cascade in turbulence
}

\author{
A Naert, R Friedrich, J Peinke
}

\section{To cite this version:}

A Naert, R Friedrich, J Peinke. Fokker-Planck equation for the energy cascade in turbulence. Physical Review E , 1997, 56 (6), pp.6719. 10.1103/PhysRevE.56.6719 . hal-01140944

\section{HAL Id: hal-01140944 \\ https://hal.science/hal-01140944}

Submitted on 11 Apr 2015

HAL is a multi-disciplinary open access archive for the deposit and dissemination of scientific research documents, whether they are published or not. The documents may come from teaching and research institutions in France or abroad, or from public or private research centers.
L'archive ouverte pluridisciplinaire HAL, est destinée au dépôt et à la diffusion de documents scientifiques de niveau recherche, publiés ou non, émanant des établissements d'enseignement et de recherche français ou étrangers, des laboratoires publics ou privés. 


\title{
Fokker-Planck equation for the energy cascade in turbulence
}

\author{
A. Naert \\ Research Institute for Electrical Communication, Tohoku University, 2-1-1 Katahira, Aoba-ku, Sendai 980-77, Japan \\ R. Friedrich \\ Institut für Theoretische Physik und Synergetik, Universität Stuttgart, D-70550 Stuttgart, Germany \\ J. Peinke \\ Experimentalphysik II, Universität Bayreuth, D-95440 Bayreuth, Germany \\ (Received 31 January 1997; revised manuscript received 5 August 1997)
}

\begin{abstract}
We present a detailed analysis of the energy dissipation averaged over a distance $r, \epsilon_{r}$, in terms of a stochastic process through scales. Using experimental data recorded in a low temperature helium jet, we give evidence that the probability density function of $\ln \left(\epsilon_{r}\right)$ obeys a Fokker-Planck equation. The drift and diffusion coefficients are calculated directly from the data. The drift is linear in $\ln \left(\epsilon_{r}\right)$ and the diffusion is constant. With these coefficients, the equation can be solved exactly, giving a Gaussian probability density function for $\ln \left(\epsilon_{r}\right)$. The mean and variance of this quantity are discussed in comparison with other log-normal models of intermittency. [S1063-651X(97)12911-7]
\end{abstract}

PACS number(s): 47.27.Gs, 47.27.Jv

The kinetic energy of a macroscopic flow must eventually be dissipated into heat. In turbulent flows, the dissipation due to molecular viscosity occurs on scales that can be much smaller than the large scale motions. Vortices of all sizes organize in such a way that their nonlinear interactions allow for a net energy flux from large to small scales. This transport process through scales is traditionally called "cascade." The first phenomenological cascade model, proposed by Kolmogorov in 1941, assumes that the energy flux through scales is constant, and thus equals the mean energy dissipation [1].

Later, it became evident that it is necessary to take into account the fluctuations of the energy flux to describe the actual turbulence, at finite Reynolds numbers Re [2]. These fluctuations cause the non-Gaussian statistics of the velocity increments, a phenomenon called intermittency $[3,4]$. In 1962, Kolmogorov and Obukhov pointed out the importance of the energy dissipation averaged over a volume of size $r$, which is approximated in the case of locally homogeneous and isotropic turbulence by

$$
\epsilon_{r}(x)=\frac{15 \nu}{r} \int_{x-r / 2}^{x+r / 2}\left(\frac{d v}{d x^{\prime}}\right)^{2} d x^{\prime},
$$

where $\nu$ denotes the kinematic viscosity and $v$ the longitudinal velocity component. Conjecturing that $\epsilon_{r}$ is a multiplicative process through scales, they invoked the central limit theorem to predict that for sufficiently small scales this quantity should have log-normal fluctuations; i.e., the fluctuations of $\ln \left(\epsilon_{r}\right)$ should be Gaussian [3]. In this theory, as well as in many others such as multifractal or shell models, it is implicitly assumed that the coupling is local in scale through the cascade process (cf. [5]).

Only recently, this hypothesis has been investigated by a direct analysis of experimental data [6]. In that work, the conditional probability density functions (PDF) of velocity increments at two different scales were evaluated, and shown to satisfy the Chapman-Kolmogorov equation. Moreover, the PDF of the velocity increments was shown to follow a Fokker-Planck equation in scales, with linear drift and quadratic diffusion coefficients in the inertial range.

In the present study we perform a similar analysis but focus our attention on the quantity $X_{r}=\ln \left(\epsilon_{r}\right)$ at different scales $r$. For convenience and without loss of generality, we use in the following the logarithmic scale $l=\ln (L / r)$, where the reference $L$ is the integral scale, the largest scale of the flow. We show that the conditional PDF of $X_{l}$ obeys the Chapman-Kolmogorov equation. Therefore $X_{l}$ is very likely to be a Markov process in $l$. We calculate the KramersMoyal coefficients that are vanishing, except the first and second. The equation governing the PDF of $X_{l}$ is then simply the Fokker-Planck equation, as for the velocity increments. The two nonvanishing coefficients, the drift and diffusion, are found to be linear and constant, respectively. With these coefficients, it is possible to solve exactly the Fokker-Planck equation, with the natural assumption that $X_{l}$ does not fluctuate at large scale. The PDF of the $X_{l}$ solution of this equation is Gaussian, the mean and variance dependence on scale are given. A peculiarity of the local energy transfer from on scale to the next one is to be asymmetric. A large decrease of $X_{l}$ is more likely to happen than a large increase. We show that this effect, which is not taken into account in the Fokker-Planck equation, does not affect significantly the Gaussian solution. Recently, a quantity called "depth of the cascade" has been introduced to characterize the intermittency of velocity increments in different flows. We show that the variance of $X_{l}$ obtained as a solution of the FokkerPlanck equation gives a precise description of this quantity over the wole inertial range, up to the largest scales of the flow.

We use a velocity sample of $10^{7}$ points, recorded in a low temperature helium jet at $\mathrm{Re}=20000, R_{\lambda}=328[7,8]$. The measurement of one component of the velocity has been performed with a cryogenic hot-wire anemometer [9] located in 


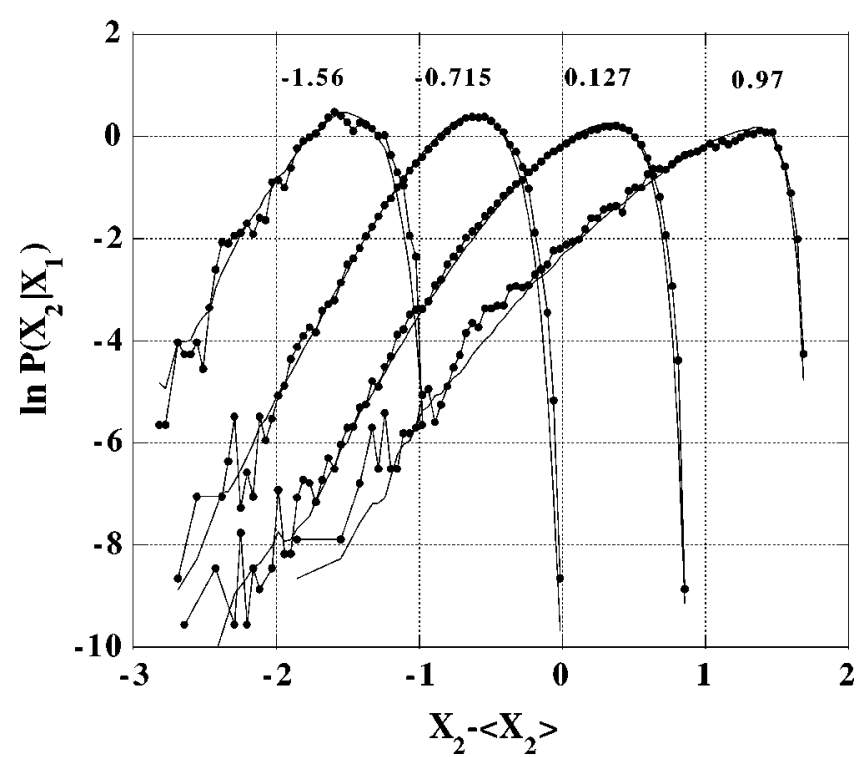

FIG. 1. Experimental verification of the Chapman-Kolmogorov equation. The conditional histogram $P\left(X_{2} \mid X_{1}\right)$ calculated directly (dotted line) and according to the right-hand side of the ChapmanKolmogorov equation (solid line) is plotted for four values of $X_{1}-\left\langle X_{1}\right\rangle$ (written above the histograms). The scales are $r_{1} / \eta=200, r_{2} / \eta=100$, and $r_{3} / \eta=142$ ( $\eta$ is the Kolmogorov viscous scale).

the center of the jet, at $10 \mathrm{~cm}$ from the $2 \mathrm{~mm}$ diameter nozzle. At this distance, the flow is completely turbulent. Using the Taylor hypothesis, $\epsilon_{l}$ has been evaluated according to Eq. (1) from the longitudinal velocity component.

Let $P\left(X_{2} \mid X_{1}\right)$ denote the conditional PDF of observing a value $X_{2}$ at scale $l_{2}$ under the condition that $X_{1}$ is realized at scale $l_{1}$. A necessary condition for a conditional PDF to describe a Markov process is the Chapman-Kolmogorov equation $[10,11]$ :

$$
P\left(X_{2} \mid X_{1}\right)=\int P\left(X_{2} \mid X_{3}\right) P\left(X_{3} \mid X_{1}\right) d X_{3}
$$

$$
\text { for any } l_{2}>l_{3}>l_{1} \text {. }
$$

This equation can be checked experimentally. We calculate the conditional histograms for sets of scales $l_{2}, l_{3}, l_{1}$. Then we compare the conditional histograms $P\left(X_{2} \mid X_{1}\right)$ calculated directly with that calculated according to the righthand side of Eq. (2). Figure 1 shows cross-sections of this histogram for several values of $X_{1}$. A good agreement is observed. Moreover, we have checked that Eq. (2) holds for any scale ratio and at all scales, down to the dissipative ones. The Chapman-Kolmogorov equation can be formulated in differential form leading to the so-called Kramers-Moyal expansion [10]:

$$
\frac{\partial}{\partial l} P\left(X_{2} \mid X_{1}\right)=\sum_{n=1}^{\infty}(-1)^{n} \frac{\partial^{n}}{\partial X_{2}^{n}} D_{n}\left(X_{2}\right) P\left(X_{2} \mid X_{1}\right) .
$$

The Kramers-Moyal coefficients are defined as

$$
D_{n}\left(X_{2}\right)=\frac{1}{n !} \lim _{l_{2} \rightarrow l_{1}} \frac{1}{l_{2}-l_{1}} \int\left(X_{1}-X_{2}\right)^{n} P\left(X_{1} \mid X_{2}\right) d X_{1} \text {. }
$$

These coefficients can be calculated directly from the data, at a finite scale ratio. We used $l_{2}-l_{1}=0.04$ (that is, $\left.r_{1} / r_{2}=1.04\right)$, below which the resolution of the velocity is no longer sufficient. Figure 2 presents $D_{1}\left(X_{l}\right)$ and $D_{2}\left(X_{l}\right)$ for several scales covering the whole inertial range. The first Kramers-Moyal coefficient is linear, and the second one is approximately constant except for small values of $\epsilon_{l}$ at small scale. In that case, the calculation is affected by the 11-bit digitalization of the velocity signal. The slope of $D_{1}$ and the value of $D_{2}$ are both independent of scale in the inertial range. We will assume that at the limit $l_{2} \rightarrow l_{1}$ :

$$
\begin{gathered}
D_{1}\left(X_{l}\right)=\gamma\left(X_{l}-\left\langle X_{l}\right\rangle\right)+F(l), \\
D_{2}\left(X_{l}\right)=D
\end{gathered}
$$

where $\left\langle X_{l}\right\rangle$ denotes the mean value of $X_{l}$. The additive function $F(l)$ is derived below from an energy conservation condition [cf. Equation (10)]. We measured from Fig. 2 $\gamma=0.21 \pm 0.02$ and $D=0.03 \pm 0.005$.

We have also evaluated $D_{4}$, which is less than $0.05\left(D_{2}\right)^{2}$. We will thus assume that $D_{4}$ vanishes in the limit $l_{2} \rightarrow l_{1}$. Pawula's theorem implies that if $D_{4}=0$, then $D_{3}$ as well as all higher order coefficients vanish [10]. The Kramers-Moyal expansion reduces to the Fokker-Plank equation, $D_{1}$ and $D_{2}$ being the drift and diffusion coefficients.

A linear drift and constant diffusion coefficients are characteristic of an Ornstein-Uhlenbeck process [10]. One may note that here, unlike the examples of Ornstein-Uhlenbeck processes usually discussed, the slope $\gamma$ of the drift is positive. As a consequence, the process is not relaxing to stationarity after a transient regime. We will see in the following that the variance monotonously increases as the cascade develops toward smaller scales.

It is natural to assume that $\epsilon_{l}$ does not fluctuate at the largest scale $l=0$, that there are no fluctuations at scales larger than the size of the system. We will thus take as the initial condition for the Fokker-Planck equation a Dirac distribution at large scale:

$$
P\left(X_{0}\right)=\delta\left(X_{0}-\ln \langle\epsilon\rangle\right)
$$

where $\langle\epsilon\rangle$ denotes the mean dissipation. The PDF of $X_{l}$ for all scales can be obtained as an exact solution of the FokkerPlanck equation [10]. It is Gaussian:

$$
P\left(X_{l} \mid X_{0}\right)=\frac{1}{\Lambda \sqrt{2 \pi}} \exp \left[-\frac{\left(X_{l}-\left\langle X_{l}\right\rangle\right)^{2}}{2 \Lambda^{2}}\right] .
$$

The mean and the variance are given by

$$
\begin{gathered}
\frac{d}{d l}\left\langle X_{l}\right\rangle=F(l), \\
\Lambda^{2}(l)=\frac{D}{\gamma}\left(e^{2 \gamma l}-1\right) .
\end{gathered}
$$



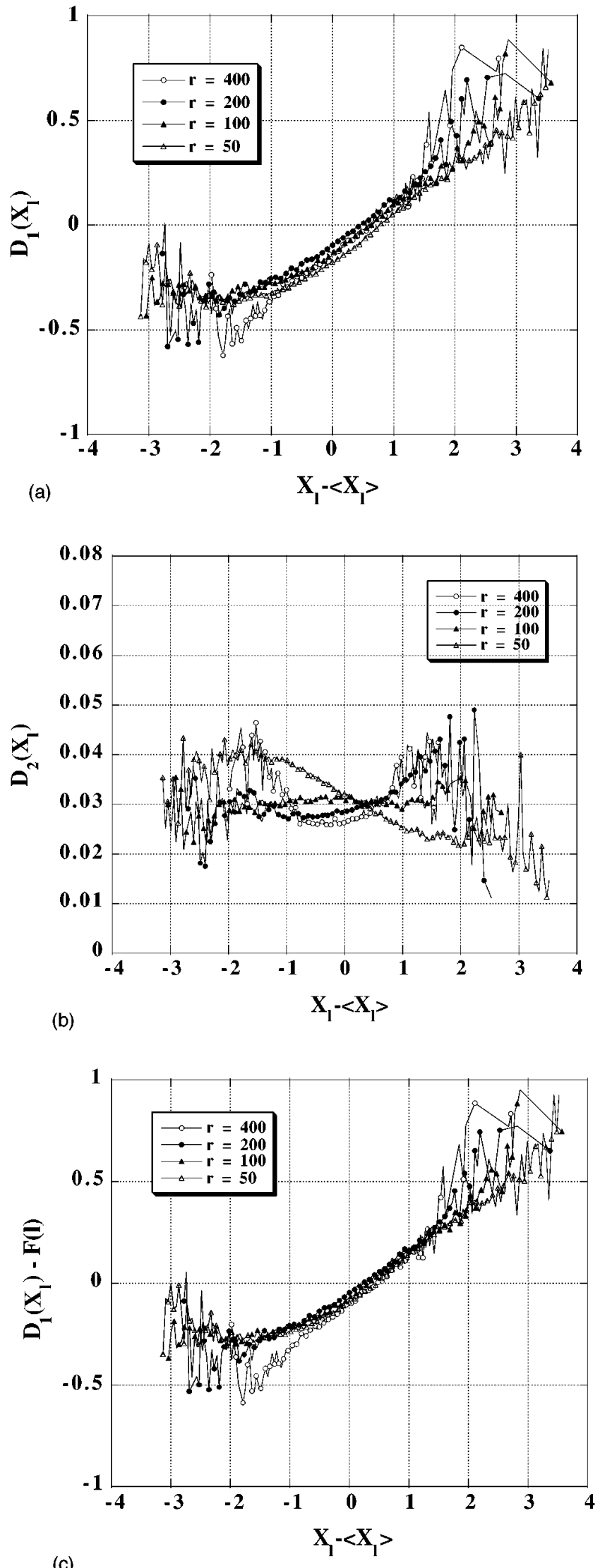

(c)

FIG. 2. Drift $D_{1}$ (a) and diffusion $D_{2}$ (b) for various values of the scale $r / \eta=400,200,100,50, \eta$ being the Kolmogorov viscous scale. (c) shows $D_{1}-F(l)$, where $F(l)$ is a function discussed in the text.

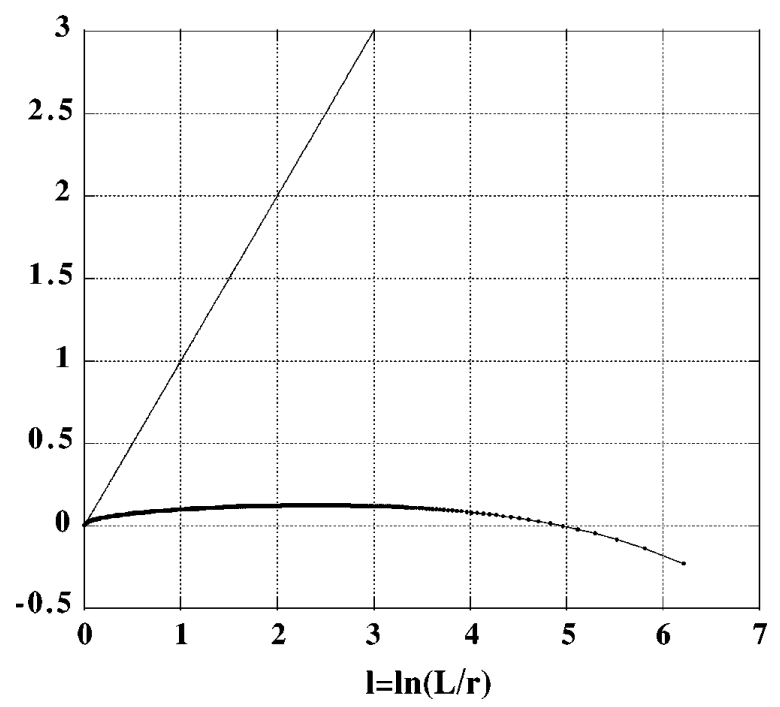

FIG. 3. A typical fluctuation amplitude $-\Lambda^{2} / 2+\Lambda / 2$ of $X_{l}-\left\langle X_{0}\right\rangle$ for all scales (dotted line), compared to the "boundary", $l=\ln (L / r)$ (solid line) vs scale $l$.

Moreover, as the energy is conserved through the cascade, the mean dissipation $\left\langle\epsilon_{l}\right\rangle$ must be independent of $l$. With the PDF given by Eq. (7), the scale dependence of $\left\langle X_{l}\right\rangle$ is easily obtained:

$$
\left\langle X_{l}\right\rangle=\left\langle X_{0}\right\rangle-\Lambda^{2} / 2 .
$$

A comparison with Eq. (8) yields

$$
F(l)=-D e^{2 \gamma l} .
$$

We show in Fig. 2(c) that the curves $D_{1}-F(l)$ for different scales collapse on a unique one. This indicates the consistence of our description of the cascade with the energy conservation condition through scales.

At this point, one may wonder about the limitation of the cascade model presented so far. We now discuss the consequence of a peculiar property of $\epsilon_{l}$ on the shape of the conditional PDF. The accessible values of $\epsilon_{l}$ are restricted by the following relationship (see also [12]):

$$
\epsilon_{2}<\epsilon_{1} e^{l_{2}-l_{1}} \quad \text { with } \quad l_{2}>l_{1},
$$

which follows directly from the definition of $\epsilon_{l}$. This constraint causes the conditional PDF to be more and more asymmetric in $X_{2}$, as $l_{1}$ is chosen closer to $l_{2}$ (see Fig. 1). Thus, the $\log$ variables $X_{l}$ always have to fulfill the condition:

$$
X_{l}<\left\langle X_{0}\right\rangle+l \text {. }
$$

An important question is whether, for large $l$, the influence of this asymmetry on the PDF is negligible or not. In other words, as this feature is not taken into account in the FokkerPlanck equation, how reliable is the Gaussian PDF solution of this equation? The fluctuations of $X_{l}$ are centered around the mean value given by Eq. (10). The sum of $\left\langle X_{l}\right\rangle$ and half of the standard deviation $\Lambda$ gives a typical fluctuation amplitude: $\left\langle X_{0}\right\rangle-\Lambda^{2} / 2+\Lambda / 2$. It can be compared to the "boundary" $\left\langle X_{0}\right\rangle+l$ of Eq. (13). In Fig. 3, we plotted 
$-\Lambda^{2} / 2+\Lambda / 2$ and $l$ for all scales. For $l$ large enough, the typical fluctuations of $X_{l}$ are always far from the region forbidden by Eq. (13). Therefore, even if the Fokker-Planck equation does not take into account local properties of the energy transfer, its Gaussian solution is a fairly good approximation. For finite $\gamma$, in the range of scales $l \gg 0, \Lambda^{2}$ can be written as

$$
\Lambda^{2}=\frac{D}{\gamma} e^{2 \gamma l}
$$

Such a dependence was predicted by Castaing et al.. [13] for a quantity $\lambda^{2}$ proportional to $\Lambda^{2}$. This important parameter $\lambda^{2}$, called "depth of the cascade," characterizes the intermittency of velocity increments [14]. It has been recently measured independently in different flows such as jets $[7,8]$, Taylor-Couette flows [15], grid flows, wind tunnel, and atmospheric boundary layer [16]. For $\operatorname{Re}>10000, \lambda^{2}$ exhibits a power law in $r$, in an intermediate range of scales. The exponent $2 \gamma$ has been measured carefully, and proven to be inversely proportional to $\ln (\mathrm{Re})$, over 4 decades of Reynolds number (see [16] for a review). At the limit of infinite Re, $\gamma$ goes to 0 . With the drift and diffusion coefficients obtained here, one can see in Fig. 4 that the variance $\Lambda^{2}$ given by Eq. (8) is related linearly to $\lambda^{2}$ calculated as in [7]. As a major improvement for the velocity statistics, Eq. (9) describes the evolution of the depth of the cascade, not only in an intermediate range of scales, but over the whole inertial range, up to the integral scale of the flow. In the viscous range of scales, i.e., for the largest values of $\Lambda^{2}$, a slight curvature is observed. This may be again a consequence of the signal digitalization, more sensible in this region of large variance, as realizations of very small $\epsilon_{l}$ are not well resolved. One may note that the curve on Fig. 4 does not pass through zero as expected. Our interpretation for this feature is that in real flows, where the Taylor hypothesis is not perfectly fulfilled, some temporal fluctuations of $\epsilon_{l}$ remain at large scale, adding a constant term on the fluctuations at all scales.

To summarize, the averaged dissipation $\epsilon_{l}$ at scale $l$ has been investigated as a stochastic process through scales. We

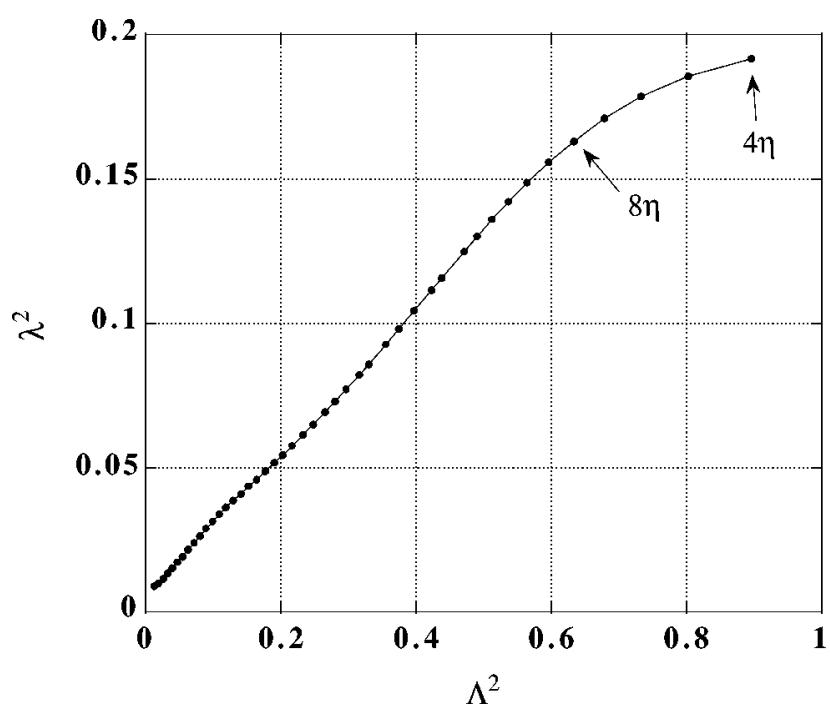

FIG. 4. The function $\lambda^{2}$ measured as in [7] plotted vs $\Lambda^{2}$ obtained from Eq. (14).

have shown that the conditional PDF of $X_{l}=\ln \left(\epsilon_{l}\right)$ fulfills the Chapman-Kolmogorov equation. The equation governing the scale dependence of this PDF is a Fokker-Planck equation, where the drift term is linear in $X_{l}$ and the diffusion coefficient is constant. For sufficiently small scales, the PDF of $X_{l}$ is Gaussian, in agreement on this point with Kolmogorov and Obukhov's model. The scaling of the velocity increments is preserved with a log-normal distribution of $\epsilon_{l}$ only if $\Lambda^{2}(l)$ is linear in $l$, as in Kolmogorov and Obukhov's model. Therefore, the expression (8), derived from the Fokker-Planck equation, is in contradiction with the existence of a pure scaling of the velocity increments. Only in the limit of infinite Reynolds number, where $\gamma \rightarrow 0$, is a pure scaling of the velocity increments.

A. Naert acknowledges financial support from the European Community and the Japanese Society for the Promotion of Science. He is grateful for the warm hospitality of Tohoku University and the University of Bayreuth. J. P. acknowledges support from the German Science Foundation (DFG).
[1] A. N. Kolmogorov, Dokl. Akad. Nauk. SSSR 32, 19, (1941) [C. R. Acad. Sci. URSS 32, 16 (1941)].

[2] A. S. Monin and A. M. Yaglom, Statistical Fluid Mechanics (The MIT Press, Cambridge, 1975).

[3] A. M. Obukhov, J. Fluid Mech. 13, 77 (1962); A. N. Kolmogorov, ibid. 13, 82 (1962).

[4] Y. Gagne, M. Marchand, and B. Castaing, J. Phys. II (France) 4, 1 (1994); A. Naert, B. Castaing, B. Chabaud, B. Hébral, and J. Peinke (unpublished).

[5] U. Frisch, Turbulence, the Legacy of A. N. Kolmogorov (Cambridge University Press, Cambridge, 1995).

[6] R. Friedrich, and J. Peinke, Physica D 102, 147 (1997); Phys. Rev. Lett. 78, 863 (1997).

[7] B. Chabaud, A. Naert, J. Peinke, F. Chillà, B. Castaing, and B. Hébral, Phys. Rev. Lett. 73, 3227 (1994).

[8] A. Naert, B. Chabaud, B. Castaing, B. Hébral, and J. Peinke (unpublished).

[9] B. Castaing, B. Chabaud, F. Chillà, B. Hébral, A. Naert, and J.
Peinke, J. Phys. III (France) 4, 671 (1994).

[10] H. Risken, The Fokker-Planck Equation (Springer, Berlin, 1984).

[11] The validity of the Chapman-Kolmogorov equation is not rigorously a proof that the process is Markovian, but it is a strong support to such a hypothesis. Non-Markovian processes fulfilling such an equation are rather rare.

[12] G. Pedrizzetti, E. A. Novikov, and A. A. Praskovsky, Phys. Rev. E 53, 475 (1996); M. Nelkin, G. Stolovitzky, ibid. 54, 5100 (1996).

[13] B. Castaing, Y. Gagne, and E. Hopfinger, Physica (Amsterdam) D 46, 177 (1990).

[14] A. Naert, L. Puech, B. Chabaud, J. Peinke, B. Castaing, and B. Hébral, J. Phys. II (France) 4, 215 (1994).

[15] F. Chillà, J. F. Pinton, and R. Labbe, Europhys. Lett. 35, 271 (1996).

[16] H. Kahalerras, Y. Malecot, Y. Gagne, and B. Castaing, Phys. Fluids (to be published). 